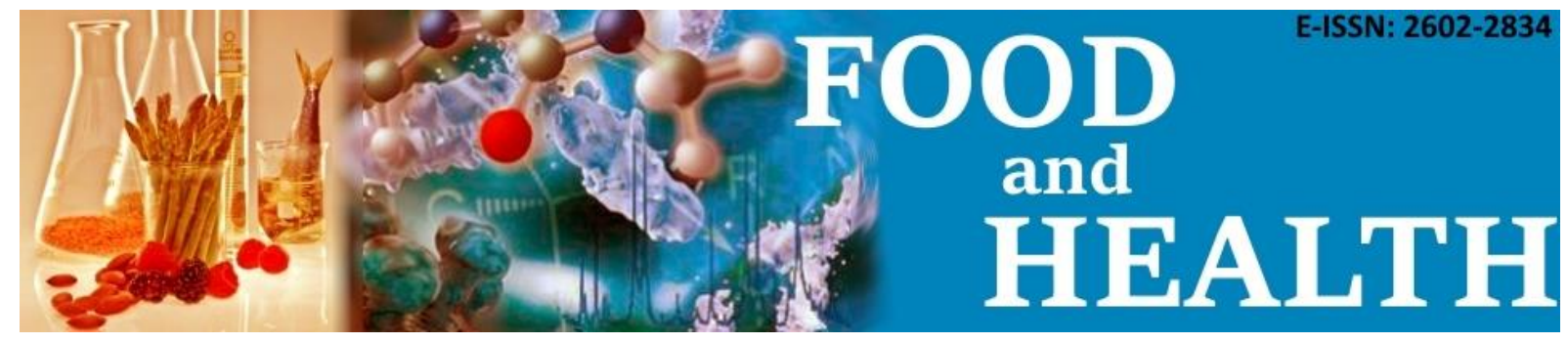

ORIGINAL ARTICLE/ORİJİNAL ÇALIŞMA

FULL PAPER

TAM MAKALE

\title{
Nigella sativa OIL COULD INDUCE OSTEOGENIC DIFFERENTIATION OF DENTAL PULP MESENCHYMAL STEM CELLS: CLINICAL NUTRITION FOR DENTISTRY
}

\author{
Ayşegül Mendi i i \\ Gazi University Faculty of Dentistry, Department of Medical Microbiology, Emek Ankara, Turkey
}

Received: 20.03 .2017

Accepted: 13.08.2017

Published online: 06.11.2017
Corresponding author:

Ayşegül Mendi, Gazi University Faculty of Dentistry, Department of Medical Microbiology, Emek Ankara, Turkey

E-mail: aysegulmendi@gmail.com

\begin{abstract}
:
A natural agent that, promotes osteogenic differentiation of dental pulp mesenchymal stem cells (DP-MSCs) could achieve success in regeneration during healing and may also prevent bone resorption and improve regeneration. We aimed to demonstrate that a Nigella sativa oil could induce differentiation of DP-MSCs. DPMSCs were isolated from 3. molars and identified with flow cytometer. Osteogenic differentiation was conducted, and calcium granules were showed by typical Alizarin Red dying and calcium concentration were determined by DICA 500. Identification results showed that the cells are mesenchymal stem cells. Alizarin Red dying signed the calcium granules and DICA 500 showed that calcium concentrations were higher than the Control Group. This preliminary study shows for the first time the inductive potential of $N$. sativa oil for osteogenic differentiation in DP-MSCs. The mechanism should be investigated in further studies.
\end{abstract}

Keywords: Dental pulp mesenchymal stem cells,

Nigella sativa, Osteogenic differentiation,

Dexamethosone

FOOD and HEALTH

E-ISSN: 2602-2834

4(1), 19-24 (2018) doi: 10.3153/JFHS18003

(C) 2015-2018 ScientificWebJournals (SWJ) 


\section{Introduction}

Dental pulp mesenchymal stem cells (DPMSCs) are a type of mesenchymal stem cell (MSCs) found in the cell-rich zone of the pulp tissue of teeth. DP-MSCs have a strong selfrenewal ability and the potential for multi-directional differentiation, which gives them great therapeutic potential for repairing damaged and/or defective tissue (Gronthos, 2000).

The presence and maintenance of alveolar bone is tooth dependent. After tooth extraction, the alveolar bone is slowly resorbed down to the body of the jaw bones. In cases of complete tooth loss, there is progressive bone resorption, which can result in extensive atrophy of the jaw bones and lead to major clinical challenges for implant placement and the construction of dental prostheses (Hoiruchi, 1999). Even without therapeutic intervention, the periodontium can exhibit a significant capacity for regeneration. However, such endogenous activity has limited capacity for periodontal regeneration. In addition, the impairment of bone formation increases in patients with osteoporosis and diabetes mellitus and related conditions (Egermann, 2005). We hpothesize that a natural agent that maintains MSCs viability, promotes osteogenic differentiation could achieve success in regeneration during healing and may also prevent bone resorption and improve regeneration. Among natural products, the seeds and oil from $N$. $s a$ tiva have attracted the interest of medical scientists as an annual herbaceous plant with black seeds. $N$. sativa is commonly known as black seeds, black cumin, black caraway seed, and Habbatul barakat, belongs to Ranunculaceae family and grows in countries bordering the Mediterranean Sea, Pakistan and India (Ali and Blunden, 2003).

The beneficial effects attributed to $N$. sativa are related to their antioxidant, antidiabetic, antihistaminic, antiepileptic, antibacterial, antitumor properties (Ali and Blunden, 2003; Kaleem et al, 2006; Kanter et al., 2006; Mabrouk et al., 2002). Furthermore, numerous studies have shown that seeds and oil from this plant are characterized by a very low de- gree of toxicity (Ali and Blunden, 2003). Thymoquinone (TQ) is the major biologically active compound of $N$. sativa which is the active ingradient for antitumor and antiiinflammatory effects (Gali-Muhtasib et al., 2005). On the other hand, TQ was shown for anabolic effects on MC3T3-E1 cells for osteogenic differentiation (Wirries et al., 2013).

Based on the aforementioned reported scientific data and considering the fact that in some cases herbal extracts and/or their oil are showing more potency than the purified components (Seeram et al., 2004, 2005), the present study was undertaken to investigate the osteogenic inductive potential of $N$. sativa oil on dental pulp mesenchymal stem cells. The study suggests that the $N$. sativa oil could be used for alternative clinical nutrition or as a mouthwash after dental treatments.

\section{Materials and Methods}

\section{Isolation and Culture of DP-MSCs}

Human dental pulp tissue was obtained from patients ( $15-20$ years of age, $n=5)$ who were undergoing extraction of their third molars for orthodontic reasons at the Department of Oral and Maxillofacial Surgery, University of Gazi, Ankara. All patients provided informed consent (Ethics Commit. Rep. No: G.Ü. B30.2. GÜN 0.21.71.00). After the tooth surfaces were disinfected, the teeth were mechanically fractured, and the dental pulp was gently isolated with forceps. The pulp tissue was rinsed in $\alpha$-MEM supplemented with $2 \mathrm{nM} \mathrm{L-}$ glutamine, $100 \mathrm{U} / \mathrm{mL}$ penicillin, $100 \mu \mathrm{g} / \mathrm{mL}$ streptomycin and $10 \%$ fetal bovine serum (FBS, Invitrogen) (hereafter referred to as the MSC culture medium), after which it was minced into fragments of 1 to $2 \mathrm{~mm}^{3}$. The tissue fragments were cultured in T75 Nunc plates in the MSC culture medium at $37^{\circ} \mathrm{C}$ in a humidified atmosphere containing $5 \% \mathrm{CO} 2$. The culture media was changed every 2 to 3 days, and the cell cultures were monitored regularly with an inverted microscope (Olympos CKX41, Tokyo, Japan). Upon reaching 70-80\% confluence, the cells were harvested with $0.05 \%$ Trypsin/EDTA (Sigma, Taufkirchen Germany) and sub-cultured for further experiments. 


\section{Food and Health, 4(1), 19-24 (2018)}

\section{Journal abbreviation: Food Health}

\section{Immunophenotypic Analysis}

The culture-expanded adherent cells were analysed by flow cytometry (BD FACSAria, USA). The antibody panel included CD29- FITC (e-bioscience, USA); CD73-PE (BD, USA), CD 90-PE (BD, USA), CD44-PE (e-bioscience, USA) as mesenchymal stromal markers, as well as their isotype controls. CD45-FITC (BD, USA); CD14PE (BD, USA); and CD34-FITC (BD USA) were used as haematopoietic markers to exclude cells of haematopoietic origin. The relative frequencies of the cells that expressed the respective surface markers were analysed using FACS Diva software 6.0.0 (BD) by acquiring 10,000 events for each sample.

\section{Effect of N. sativa oil on DP-MSCs Osteogenic Differentiation}

$N$. sativa oil was purchased from market in Ankara, Turkey. A concentration of $1 \%$ was prepared in osteogenic and adipogenic differentiation media (Pittenger et al., 1999). The images of differentiation were obtained at 21 day with a CKX41 digital imaging microscope (Olympus, Tokyo, Japan). The calcium ion concentration in the differentiation medium was measured using a QuantiChrom calcium assay kit according to the manufacturer's instructions (DICA 500, BioAssay Systems, Hayward, USA).

\section{Results and Discussion}

\section{Identification of MSCs}

The common MSC markers (CD29, CD73, CD44, and CD90) were constitutively positive (>95\%) and the hematopoietic markers (CD14, CD34, and CD45) were negative (>95) in all samples tested, indicating a mesenchymal origin of the cells (Figure 1). Interestingly we found two subpopulations in the flow cytometer analysis suggesting that dental pulp has multiple stem cell niches. This result was found in compliance with Pisciotat et al (2015) showing the heterogeneity of the stem cell population residing within the human dent al pulp, particularly its peculiar embryological origin, might explain the existence of two different subpopulations. Of course in further studies, subpopulations would be sorted and analyzed.
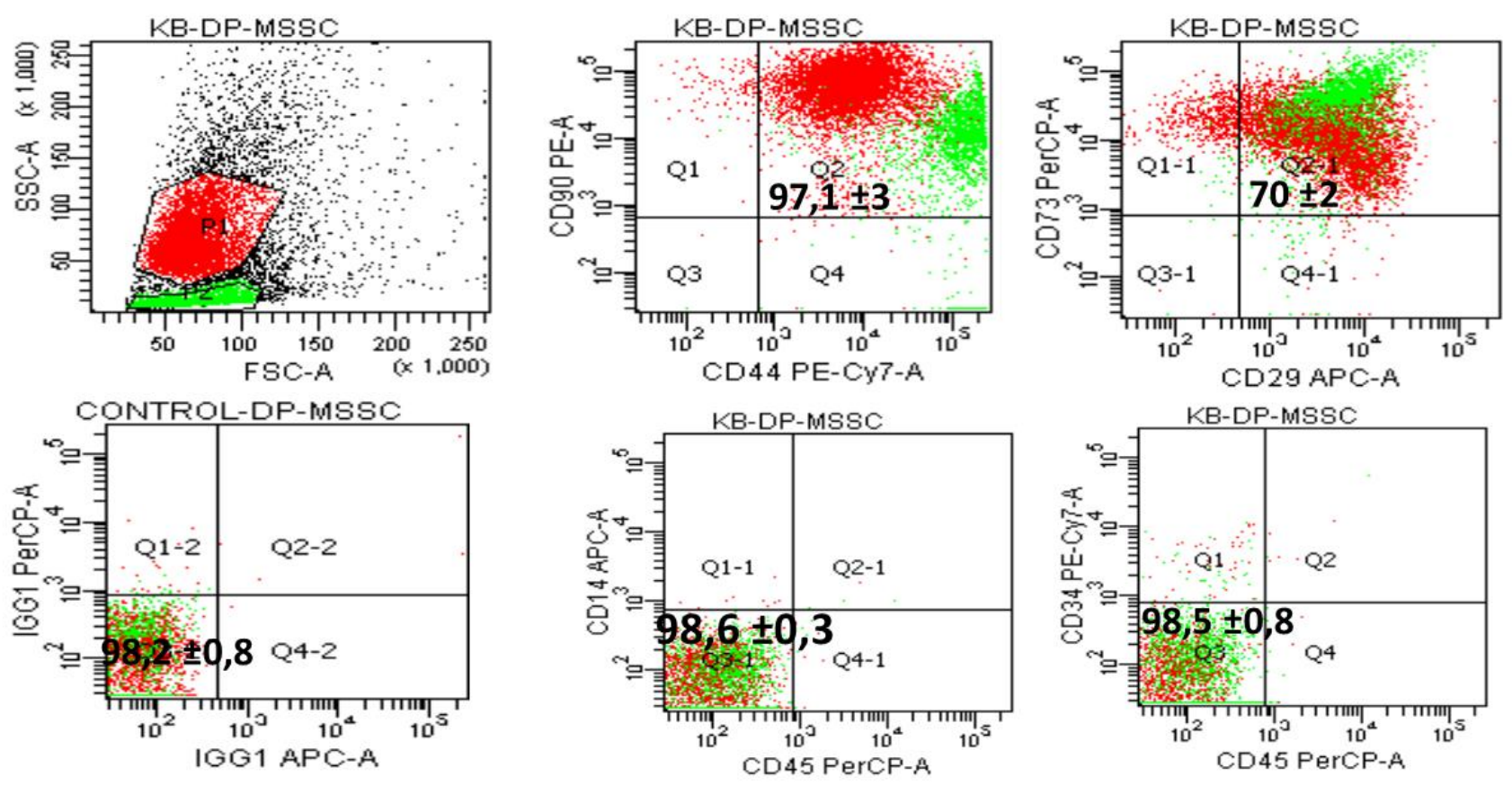

Figure 1. Surface markers of DP-MSCs. The cells were positive for CD90, CD44, CD29 and CD73 (the mesenchymal stem cell markers) and negative for CD14, CD45, and CD34 (the hematopoietic stem cell markers). 


\section{Food and Health, 4(1), 19-24 (2018)}

\section{Journal abbreviation: Food Health}

\section{Differentiation Assays of MSCs}

The characteristics features of the cells were studied. (Figure 2). Approximately $20 \%$ of the cells became rounder; however, no lipid droplets were observed in DP-MSCs for adipogenic differentiation. In contrast to adipogenic differentiation, the DP-MSCs underwent rapid osteogenic differentiation. Calcium granules similar to bone nodules were seen in $N$ sativa treated group. We also determined the calcium concentration (Figure 3 ).

The osteogenic differentiation potentials of DPMSCs in vitro and in vivo have been well documented in a variety of studies (Gronthos et al., 2000; D'aquino et al., 2007). Adipogenic differentiation was not seen in DP-MSCs. Our findings were agree with those of Gronthos et al (2000) who expanded DP-MSCs from single-cell clones and demonstrated that they exhibited osteogenic differentiation and did not form lipid-laden adipocytes. $N$. sativa treated cells showed increased ostegenic differentiation. Calcium granules were clear and compact which was a sign of well differentiation.

In dentistry, $N$. sativa showed a lowered caries score and plaque index (Shaker et al., 2014). Studies comparing the oil or TQ exhibited that $N$. $s a$ tiva essential oil has more strong activity against Streptococcus mitis, Staphylococcus mutans than the pure TQ (Harzallah et al., 2011). The N. sativa oil was found effective in inhibiting the adherence of $S$. mutans to the tooth surface at $10 \%$ concentration (Abd-Awn et al., 2012) that we used for osteogenic differentiation assays. Also the oral administration of TQ helped in periodontal disease prevention as it diminishes alveolar bone resorption (Özdemir et al., 2012). This is the first study showing dental pulp mesenchymal stem cell osteogenic differentiation with $N$. sativa. Of course there should be further studies investigating the mechanism of increased differentiation. Nevertheless the obtained data suggest that $N$. sativa oil could be used as an alternative agent for dental bone regeneration studies.

\section{DENTAL PULP MSCs}

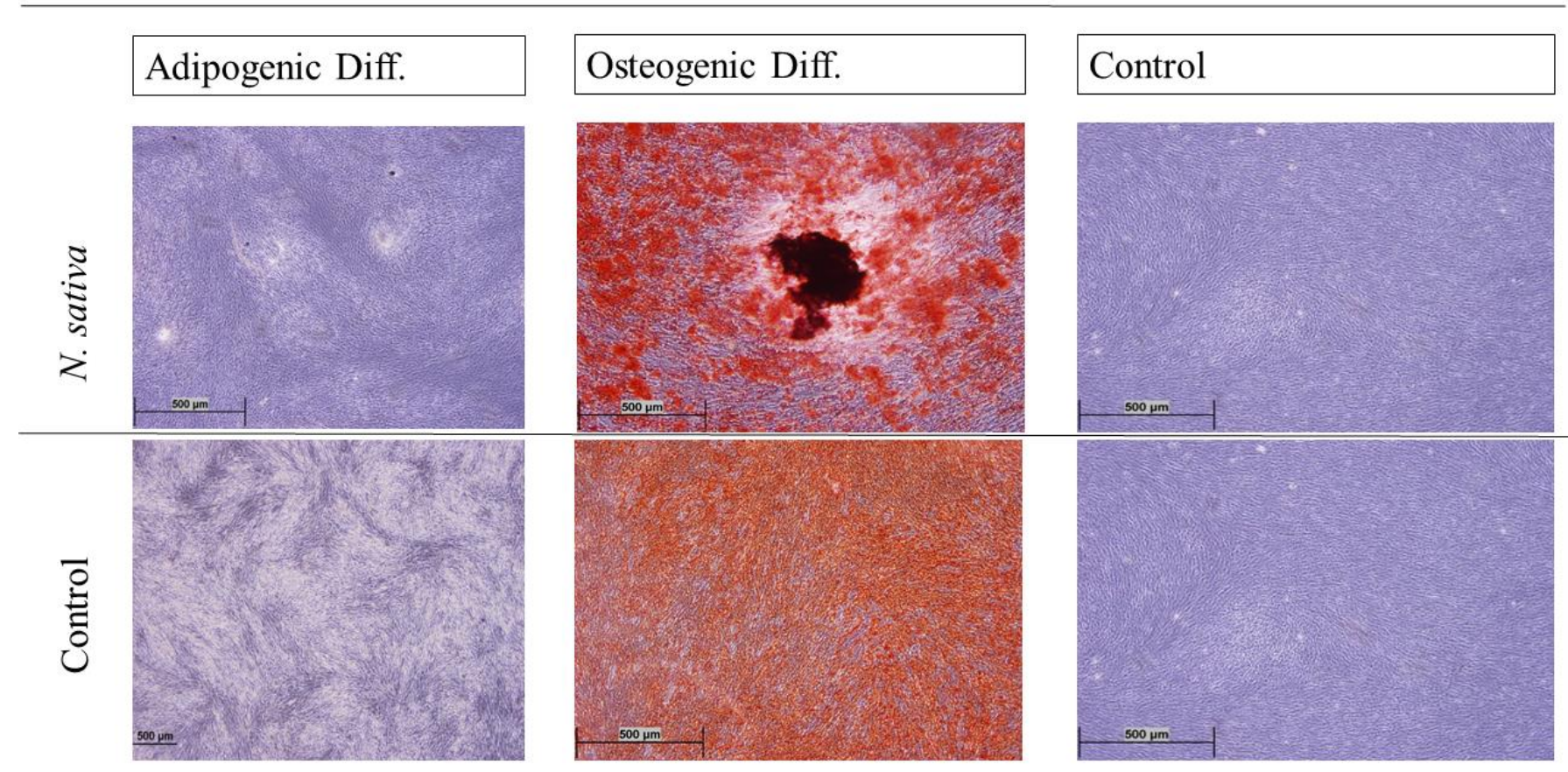

Figure 2. Differentiation potential of DP-MSCs is shown as Control Group. Adipogenic differentiation was not shown in DP-MSCs in both Control and N. sativa Group. Osteogenic differentiation was typically observed in Control Group with calcium granules and extracellular matrix. Calcium granules are seen as black nodules in $N$. sativa Group and the extracellular matrix was dyed red. (4x, Olympos CKX41,Tokyo, Japan). 


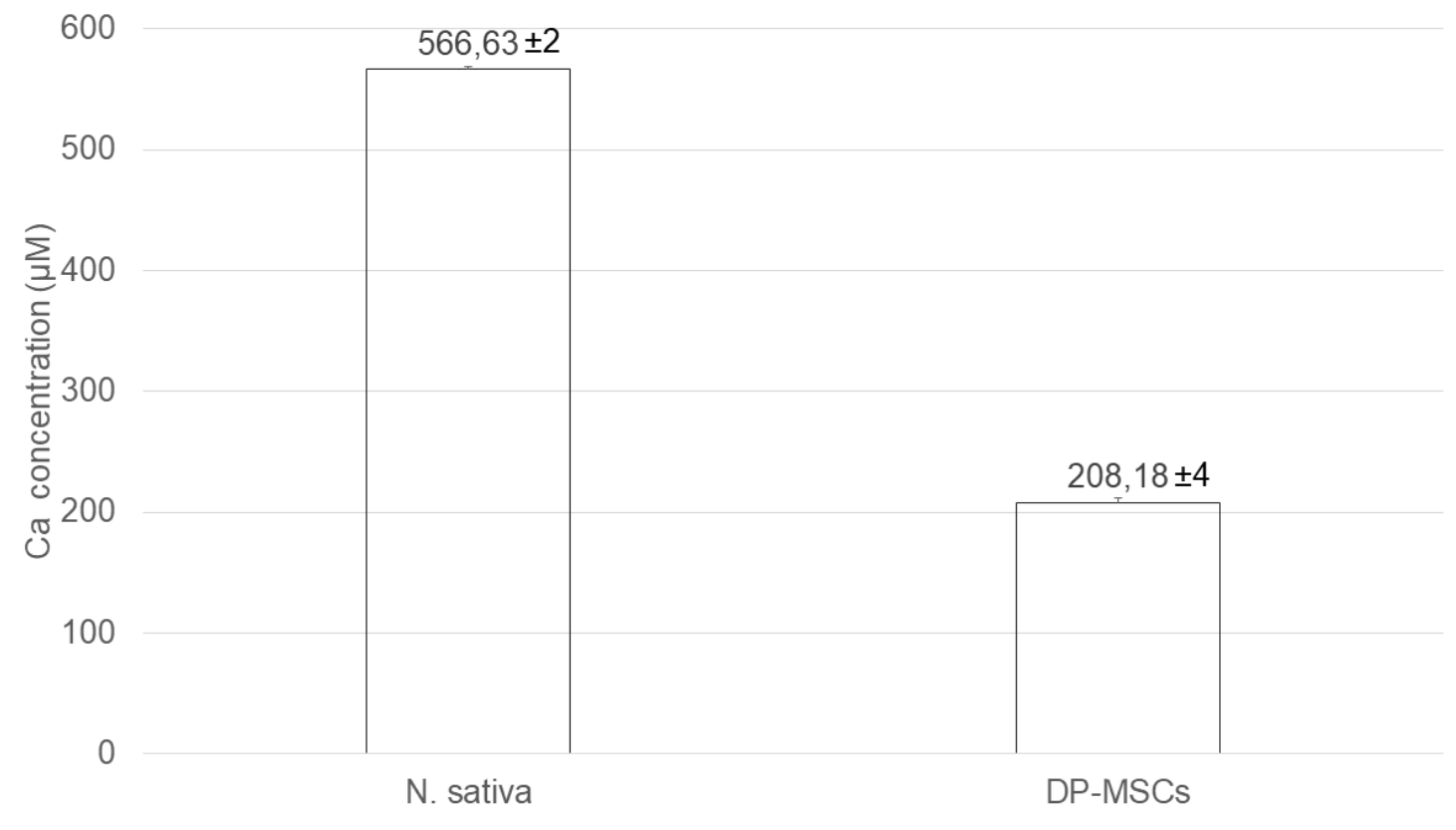

Figure 3. Calcium concentration of DP-MSCs was detected by Quantichrome calcium assay Kit. Calcium concetration was found increased in DP-MSCs treated with $N$. sativa.

\section{Conclusion}

There are number of people suffering from implant failure and/or reduced alveolar bone regeneration etc. There is consequently a growing need for therapies that provide a balance between bone resorption and bone formation and of course, increasing the strength of bone and having least side effects. Therefore, a closer look for plant based agents is needed. Here, N. sativa could be used safely after dental treatments as an adjunct therapy. Besides immunotherapy and antioxidant studies animal models with mesenchymal stem cells should be used to test the effects on periodontal regeneration especially.

\section{Acknowledgement}

This study was supported by the Turkish Scientific and Technological Research Council (TUBITAK), Project no: SBAG 113S448. I specially thank to Prof. Dr. Derviş Yılmaz for assistance and encouragement in dental research studies.

\section{Conflict of Interests}

Author declare that there is no conflict of interests.

\section{References}

Abd-Awn, B., Al-Dhaher, Z. \& Al-Dafaai, R. (2012). The effect of black seed oil extracts on mutans streptococci in comparison to chlorhexidine gluconate (in vitro). Journal of Baghdad College of Dentistry, 24, 126-131.

Ali, B.H. \& Blunden, G. (2003). Pharmacological and toxicological properties of Nigella sativa. Phytother. Res. 17, 299-305.

Kirui, P.K., Cameron, J., Benghuzzi, H.A., Tucci, M., Patel, R., Adah, F. \& Russell, G. (2004). Effects of sustained delivery of thymoqiunone on bone healing of male rats. $B i$ omedical Science Instrumentation, 40, 111116.

D'aquino, R., Graziano, A., Sampaolesi, M., Laino, G., Pirozzı, G., De Rosa, A. \& Papaccio, G. (2007). Human postnatal dental pulp cells co-differentiate into osteoblasts and endotheliocytes: a pivotal synergy leading to adult bone tissue formation. Cell Death \& Differentiation. 14, 1162-1171.

Egermann, M., Goldhahn, J. \& Schneider, E. (2005). Animal models for fracture treatment in osteoporosis. Osteoporos International, 16(suppl2), 129-138. 


\section{Food and Health, 4(1), 19-24 (2018)}

\section{Journal abbreviation: Food Health}

Gali-Muhtasib, H., Roessner, A. \& SchneiderStock, R. (2005). Thymoquinone: a promising anti-cancer drug from natural sources. The International Journal of Biochemistry \& Cell Biology, 38, 1249-1253.

Gronthos, S., Mankani, M., Brahim, J., Robey, P.G. \& Shi, S. (2000). Postnatal human dental pulp stem cells (DPSCs) in vitro and in vivo. Proceedings of the National Academy of Science, 97, 13625-13630.

Harzallah, H., Kouidhi, B., Flamini, G., Bakhrouf, A. \& Mahjoub, T. (2011). Chemical composition, antimicrobial potential against cariogenic bacteria and cytotoxic activity of Tunisian Nigella sativa essential oil and thymoquinone. Food Chemistry, 129, 1469-1474.

Hoiruchi, K., Amizuka, N., Takeshita, S., Takamatsu, H., Katsuura, M., Ozawa, H., Toyama, Y., Bonewald, L.F. \& Kudo, A. (1999). Identification and chracterization of a novel protein, perostin, with restricted expression to periosteum and periodontal ligament and increased expression by transforming growth factor beta. Journal of Bone \& Mineral Research, 14,1239-1249.

Kaleem, M., Kirmani, D., Asif, M., Ahmed, Q., Bano, B. (2006). Biochemical effects of Nigella sativa L. seeds in diabetic rats. Indian Journal of Experimental Biology, 44, 745748.

Kanter, M., Coşkun, O. \& Uysal, H. (2006). The antioxidative and antihistaminic effect of $\mathrm{Ni}$ gella sativa and its major constituent, thymoquinone on ethanol-induced gastric mucosal damage. Archves of Toxicology, 80, 217-224.
Mabrouk, G.M., Moselhy, S.S., Zohny, S.F., Ali, E.M., Helal, T.E., Amin, A.A. \& Khalifa, A.A. (2002). Inhibition of methylnitrosourea (MNU) induced oxidative stress and carcinogenesis by orally administered bee honey and Nigella grains in Sprague Dawley rats. Journal of Experimental and Clinical Cancer Research, 21, 341-346.

Pisciota, A., Carnevale G., Meloni, S., Ricio, M., Biasi, S.D., Gibellini, L., Ferrari, A., Bruzzesi, G. \& de Pol, A. (2015). Human dental pulp stem cells (hDPSCs): isolation, enrichment and comparative differentiation of two sub-populations. BMC Developmental Biology, 15, 14-16

Wirries, A., Schubert, A.K., Zimmermann, R., Jabari, S., Ruchholtz, S. \& El-Najjar, N. (2013). Thymoquinone accelerates osteoblast differentiation and activates bone morphogenetic protein-2 and ERK pathway. International Immunopharmacology, 15(2), 381-386.

Ozdemir, H., Kara, M.I., Erciyas, K., Ozer, H. \& Ay, S. (2012). Preventive effects of thymoquinone in a rat periodontitis model: a morphometric and histopathological study. Journal of Periodontal Research, 47, 74-80.

Pittenger, M.F., Mackay, A.M., Beck, S. (1999). Multilineage potential of adult human mesenchymal stem cells. Science, 284, 1168-1170.

Shaker, A. \& Al-Wafi, H. (2014). Benefits of thymoquinone, a Nigella sativa extract in preventing dental caries initiation and improving gingival health. ProQuest LLC, 72. 